\title{
Attractive Energy and Entropy or Particle Size: the Yin and Yang of Physical and Biological Science
}

\author{
Douglas HENDERSON* \\ (Department of Chemistry and Biochemistry, Brigham Young University, Provo UT 84602, USA)
}

Erratum to Interdiscip Sci Comput Life Sci (2009) 1: 1-11

DOI: $10.1007 / \mathrm{s} 12539-008-0001-3$

The original version of this article unfortunately contained a mistake. Table 1 was missing in the paper version and the HTML version of this article. The missing table is given below.

Table 1 Equation of state of methyl chloride $T_{c}=$ $143.1{ }^{\circ} \mathrm{C}, p_{c}=65.919 \mathrm{~atm} V_{c}=2.755 \mathrm{~cm}^{3} / \mathrm{gm}$

\begin{tabular}{cccc}
\hline $\begin{array}{c}T \\
\left({ }^{\circ} \mathrm{C}\right)\end{array}$ & $\begin{array}{c}V \\
\left(\mathrm{~cm}^{3} / \mathrm{gm}\right)\end{array}$ & $\begin{array}{c}p(\operatorname{expt} 1) \\
(\mathrm{atm})\end{array}$ & $\begin{array}{c}p(\mathrm{vdW}) \\
(\mathrm{atm})\end{array}$ \\
\hline \multirow{2}{*}{125} & 88.266 & 6.975 & 7.063 \\
& 27.774 & 20.049 & 20.629 \\
& 16.333 & 30.664 & 32.017 \\
& 11.070 & 39.817 & 42.215 \\
& 7.204 & 50.015 & 52.848 \\
& 67.069 & 9.567 & 9.632 \\
& 18.099 & 30.398 & 31.288 \\
& 8.321 & 51.077 & 53.818 \\
& 1.939 & 69.954 & 251.9 \\
& 1.610 & 101.510 & 936.55 \\
& 1.400 & 206.85 & 4265. \\
\hline
\end{tabular}

The online version of the original article can be found at http://dx.doi.org/10.1007/s12539-008-0001-3.

\footnotetext{
*Corresponding author.

E-mail: doug@chem.byu.edu
} 